\title{
Role of cerium in lithium niobate for holographic recording
}

\author{
Xuefeng Yue, A. Adibi, T. Hudson, ${ }^{\text {a) }}$ K. Buse, and D. Psaltis ${ }^{\text {b) }}$ \\ California Institute of Technology, Department of Electrical Engineering, MS 136-93, Pasadena, \\ California 91125
}

(Received 8 October 1999; accepted for publication 29 November 1999)

\begin{abstract}
Cerium-doped lithium niobate crystals are tested for holographic recording. A photochromic effect is observed in crystals doped with cerium and manganese. But two-center recording in the sample is not as effective as in iron and manganese doubly doped crystals. Photocurrent measurements in cerium and iron singly doped crystals indicate that the photovoltaic constant in the cerium-doped crystal is only one third of that of the iron-doped one. This is the main reason accounting for the low sensitivity of cerium-doped lithium niobate crystals. However, in the diffusion dominated case, i.e., for reflection geometry, cerium-doped lithium niobate may give a strong effect. (C) 2000 American Institute of Physics. [S0021-8979(00)08505-4]
\end{abstract}

\section{INTRODUCTION}

Holographic data storage is a promising candidate for the next-generation mass-storage system. It offers two substantial advantages over conventional storage technologies: (1) multiple pages of data can be stored in the same volume, (2) many bits are recalled in parallel. This enables a storage device that has potentially a high storage density and data transfer rate. ${ }^{1}$ Inorganic photorefractive oxide crystals such as lithium niobate $\left(\mathrm{LiNbO}_{3}, \mathrm{LN}\right)$ have attracted much attention in the past due to their reversibility, moderate sensitivity, and availability in large size and good quality. ${ }^{1-4}$ The storage of volume phase holograms in $\mathrm{LiNbO}_{3}$ relies on the presence of localized centers containing electrons that can be optically excited into the conduction band. Various transition metals have been introduced into $\mathrm{LiNbO}_{3}$ for the improvement of sensitivity and dynamic range. ${ }^{5,6}$ Among various dopands, $\mathrm{Fe}$ is most effective in producing large improvements in both the sensitivity and the maximum diffraction efficiency. Recently two-center recording in $\mathrm{Fe}$ and $\mathrm{Mn}$ doubly doped $\mathrm{LiNbO}_{3}$ has been realized to achieve persistent storage. ${ }^{7}$ Illumination with ultraviolet light excites electrons from deep center $(\mathrm{Mn})$ to the conduction band and some of them may get trapped at the shallower center $(\mathrm{Fe})$, which permits the storage of data by using red light (wavelength $633 \mathrm{~nm}$ ), but no erasure during the subsequent readout in the absence of ultraviolet light. For the improvement of this technique, a more red-sensitive dopand, which can replace $\mathrm{Fe}$, is very important.

Cerium is known to be an effective trap center, which can provide and capture charge carriers, in different kinds of photorefractive crystals including strontium-barium-niobate $(\mathrm{SBN})^{8}$ and barium-titanate $\left(\mathrm{BaTiO}_{3}\right){ }^{9,10} \mathrm{Ce}$-doped SBN has a very high sensitivity at visible wavelength, ${ }^{8}$ and Cedoped $\mathrm{BaTiO}_{3}$ is sensitive to both visible and near infrared light. ${ }^{9,10}$ Some previous reports on $\mathrm{Ce}$ and $\mathrm{Fe}$ doubly doped $\mathrm{LiNbO}_{3}$ suggest that this material has a wide spectral re-

\footnotetext{
a) Also with: U.S. Army Aviation and Missile Command, Redstone Arsenal, AL 35898-5248.

${ }^{b)}$ Author to whom correspondence should be addressed.
}

sponse and shows higher sensitivity. ${ }^{11,12}$ Charge transport from and to Fe centers is already well known, but the impact of $\mathrm{Ce}$ doping on the buildup of space-charge fields in $\mathrm{LiNbO}_{3}$ is still unclear. McMillen et al. have reported holographic recording in specially doped $\mathrm{LiNbO}_{3}$ crystals including a Ce singly doped one, ${ }^{13}$ but no systematic consideration for the role of cerium has been carried out. In our present work, we have found that the $\mathrm{Ce}$ and $\mathrm{Mn}$ doubly doped $\mathrm{LiNbO}_{3}$ has a strong photochromic effect, which may be very attractive for two-center persistent data storage. In this contribution, we investigate the photorefractive performance of Ce-doped $\mathrm{LiNbO}_{3}$ crystals. Comparisons will be made among the nominally pure, $\mathrm{Ce}-$, Fe-, and Mn-doped samples.

\section{SAMPLES}

Singly doped $(\mathrm{Ce}, \mathrm{Fe}$, and $\mathrm{Mn}$ ) and doubly doped (Ce:Mn, Fe:Mn) $\mathrm{LiNbO}_{3}$ samples as well as a nominally pure one are used in present experiments. Notations, doping levels, and dimensions of the samples are listed in Table I. All doping levels refer to the values introduced into the melt. It is known that even in nominally pure crystals there are usually some background impurities incorporated, ${ }^{14}$ but the concentrations of such background impurities are generally much smaller than the intentionally doped impurity levels.

All samples are $y$ cut and polished to optical quality. Two kinds of thermal treatments have been conducted: oxidation and reduction. During oxidation, the samples are kept in an oven with oxygen atmosphere at $1000^{\circ} \mathrm{C}$ for at least 12 $\mathrm{h}$, while they are heated to $1000^{\circ} \mathrm{C}$ in argon atmosphere for $12 \mathrm{~h}$ during reduction. In this way, the absorption of the doped samples can be significantly changed, i.e., the valence states of the trap centers can be varied.

\section{EXPERIMENTS AND RESULTS}

As mentioned above, the main purpose of this work is to test whether $\mathrm{Ce}$ is an effective trap center in lithium niobate crystals and to measure the performance of Ce-doped crystals for holographic recording, especially in the red. So we conducted measurements including conventional holographic 
TABLE I. Description of the samples used in the experiments. The doping concentrations are wt $\%$ of the oxide $\left(\mathrm{Fe}_{2} \mathrm{O}_{3}, \mathrm{Ce}_{2} \mathrm{O}_{3}\right.$, and $\left.\mathrm{MnO}\right)$ in the melt and dimensions are $a \times b$ (thickness) $\times c$ in $\mathrm{mm}^{3}$.

\begin{tabular}{lllc}
\hline \hline Notation & \multicolumn{1}{c}{ Dopant } & Doping level & Dimensions \\
\hline LN & normally pure & - & $5 \times 5 \times 7$ \\
Ce:LN & $\mathrm{Ce}$ & $0.01-0.02$ & $5 \times 5 \times 7$ \\
Fe:LN & $\mathrm{Fe}$ & $0.01-0.02$ & $5 \times 5 \times 7$ \\
Mn:LN & $\mathrm{Mn}$ & 0.05 & $4 \times 1 \times 6$ \\
Ce:Mn:LN & $\mathrm{Ce}$ & 0.085 & $10 \times 2 \times 10$ \\
& $\mathrm{Mn}$ & 0.01 & \\
Fe:Mn:LN & $\mathrm{Fe}$ & 0.085 & $10 \times 2 \times 10$ \\
& $\mathrm{Mn}$ & 0.01 & \\
\hline \hline
\end{tabular}

recording in singly doped samples, sensitization by ultraviolet light, and two-center recording as well as bulk photovoltaic current measurements.

\section{A. Photochromic effect}

It is known that the crystals are often photochromic if two kinds of trap centers exist between the valence and conduction bands, which means that the absorption can be changed by illumination because of the redistribution of charge carriers between these different traps. Three samples, i.e., Mn:LN, Fe:Mn:LN, and Ce:Mn:LN are used in sensitizing measurements. All crystals are strongly oxidized before the sensitization measurements were started to ensure that most of electrons are removed from the shallower trap centers $(\mathrm{Ce}$ or $\mathrm{Fe})$.

In our experiment, we use ultraviolet light (wavelength $404 \mathrm{~nm}$ and intensity $4 \mathrm{~mW} / \mathrm{cm}^{2}$ ) to illuminate the samples. A very weak red beam $(633 \mathrm{~nm})$ is used to probe the transmission as a function of illuminating time. Figure 1 shows the variation of the normalized transmission versus time for different samples. The relative change of transmission in Ce:Mn:LN is much larger than that in Fe:Mn:LN. We perform the sensitization measurement with the Mn singly doped sample, too. As it can be seen from Fig. 1, no change in absorption can be observed in this case, which means that the absorption variation in Ce:Mn: LN can be attributed to

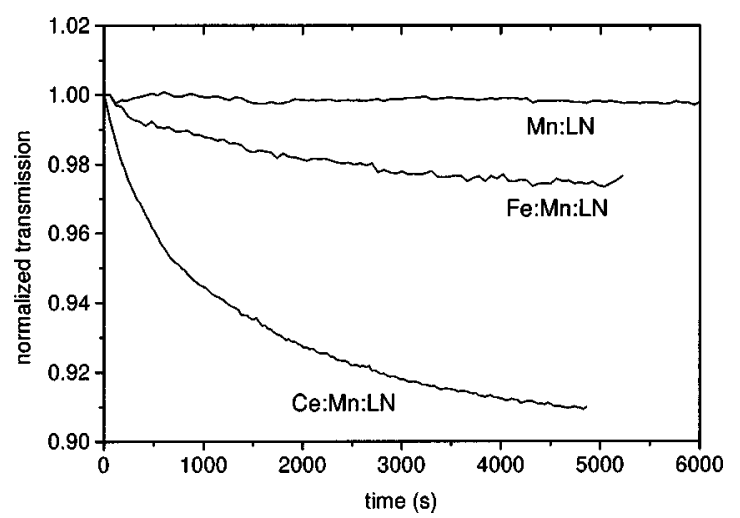

FIG. 1. Variation of normalized transmission at $633 \mathrm{~nm}$ for variously doped $\mathrm{LiNbO}_{3}$ during illumination with ultraviolet light. Wavelength and intensity of the ultraviolet light are $404 \mathrm{~nm}$ and $4 \mathrm{~mW} / \mathrm{cm}^{2}$, respectively. The samples were first strongly oxidized to ensure that most of the shallower centers are emptied.

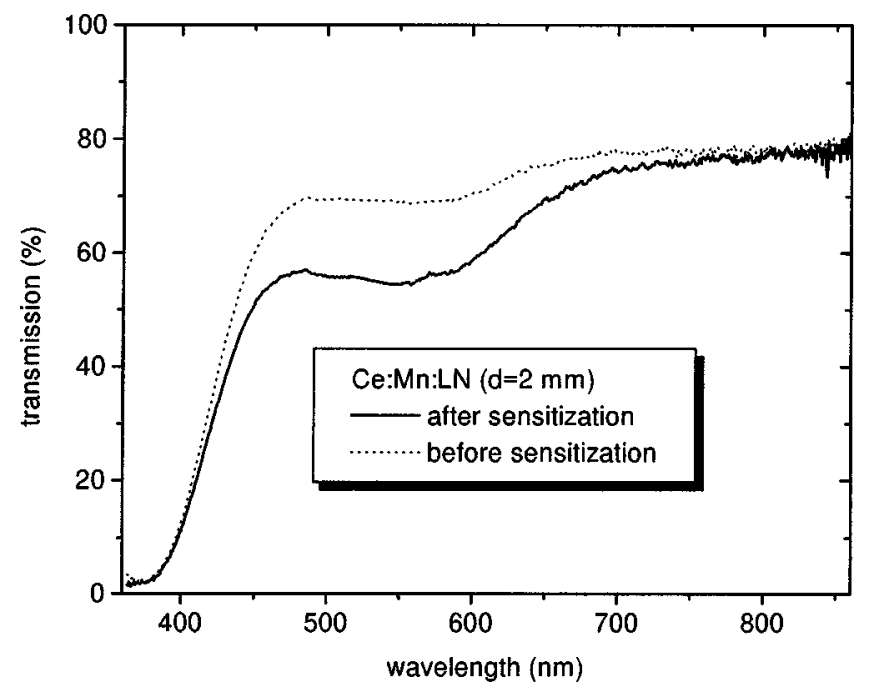

FIG. 2. Transmission spectra of the $\mathrm{Ce}$ and $\mathrm{Mn}$ doubly doped sample (Ce:Mn:LN) before (dotted curve) and after (solid curve) ultraviolet illumination. Wavelength and intensity of the ultraviolet light are $404 \mathrm{~nm}$ and 4 $\mathrm{mW} / \mathrm{cm}^{2}$, respectively, and the illumination time is $2 \mathrm{~h}$.

the filling of Ce traps with the help of ultraviolet light and not to some kinds of emptied background impurities.

The transmission spectra before and after ultraviolet illumination in Ce:Mn:LN are measured and the results are presented in Fig. 2. It can be seen that after $2 \mathrm{~h}$ illumination by ultraviolet, there is a broad induced absorption in the range from 450 to $650 \mathrm{~nm}$. The large absorption caused by ultraviolet makes the material promising for two-center recording at red.

\section{B. Sensitivity}

A He-Ne laser operating at $633 \mathrm{~nm}$ is used for holographic recording. The total intensity $I_{0}$ of the writing beams is $26 \mathrm{~mW} / \mathrm{cm}^{2}$. Both writing beams are ordinarily polarized and impinge on the crystal symmetrically at an incident angle of $23^{\circ}$ in air. The grating vector of the interference pattern is aligned along the crystallographic $c$ axis. The diffraction efficiency $\eta$ is defined as the ratio between the diffracted and incident beam intensities, while the recording sensitivity is defined by $S=\left.(\partial \sqrt{\eta} / \partial t)\right|_{t=0} /\left(I_{0} d\right)$, where $d$ is the thickness of the sample.

First we strongly reduce the samples including LN, Ce:LN, Ce:Mn:LN, and Fe:LN. In Fig. 3 we show the recording and erasure curves obtained in nominally pure, $\mathrm{Ce}$ doped and Fe-doped samples. All of the samples have the same thickness. From these measurements, we get the sensitivity data: $\quad S(\mathrm{LN})=0.002 \mathrm{~cm} / \mathrm{J}, S(\mathrm{Ce}: \mathrm{LN})=0.007 \mathrm{~cm} / \mathrm{J}$, $S($ Ce:Mn:LN $)=0.020 \mathrm{~cm} / \mathrm{J}, S(\mathrm{Fe}: \mathrm{LN})=0.033 \mathrm{~cm} / \mathrm{J}$. Clearly, the increase of Ce concentration leads to an increase of recording sensitivity. Mn traps are too deep to get involved in holographic recording in red. Therefore, holographic recording in Ce:Mn: $\mathrm{LN}$ is similar to a $\mathrm{LiNbO}_{3}$ crystal doped with only $0.085 \mathrm{wt} \% \mathrm{Ce}$, corresponding to $\mathrm{Ce}$ concentration of $C_{\mathrm{Ce}}=14 \times 10^{24} \mathrm{~m}^{-3}$. This is two times as large as the concentration in Fe: $\mathrm{LN}\left(C_{\mathrm{Fe}} \leqslant 7 \times 10^{24} \mathrm{~m}^{-3}\right)$. However, the sensitivity in Ce:Mn:LN is still smaller than that in Fe:LN. 


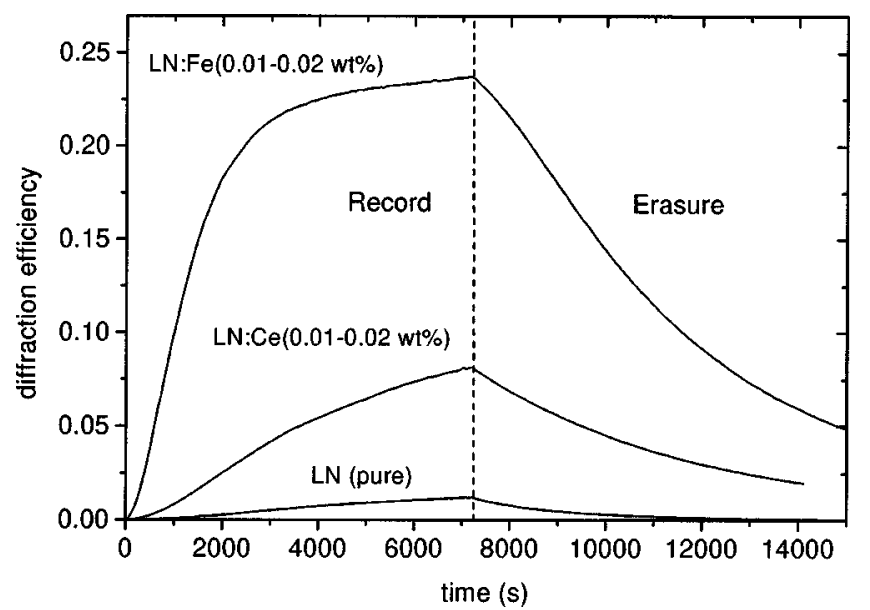

FIG. 3. Evolution of the diffraction efficiency during holographic recording and erasure in nominally pure, Ce-doped and Fe-doped $\mathrm{LiNbO}_{3}$. The three samples have the same dimensions with the thickness $d=5 \mathrm{~mm}$ (wavelength of writing beam $633 \mathrm{~nm}$, total intensity $26 \mathrm{~mW} / \mathrm{cm}^{2}$, ordinary polarized, grating vector along $c$ axis, and grating spacing $0.8 \mu \mathrm{m}$ ).

\section{Two-center recording}

The large light-induced absorption at $633 \mathrm{~nm}$ caused by ultraviolet light in Ce:Mn:LN is a very promising aspect for two-center recording, but the smaller sensitivity of holographic recording in Ce-doped $\mathrm{LiNbO}_{3}$ compared to that in the Fe-doped sample is a discouraging result. To be certain whether Ce:Mn:LN is a good alternative to Fe:Mn:LN, we conduct two-center recording in Ce:Mn:LN.

Two-center recording in Ce:Mn:LN is carried out by the following procedure: the strongly oxidized sample is first illuminated by incoherent ultraviolet light for about $2 \mathrm{~h}$, then two ordinarily polarized red beams are turned on with the ultraviolet light still present. After $3 \mathrm{~h}$ recording, the ultraviolet light and one of the red beams are turned off. The diffracted beam intensity is monitored for about $10 \mathrm{~h}$. As shown in Fig. 4, the erasure of the recorded grating consists of two parts: a fast decay and a relatively slow decay. These are typical characteristics of two-center recording. ${ }^{7}$ The same procedure is conducted for Fe:Mn:LN. A maximum diffraction efficiency of $25 \%$ is reached in this case, which is approximately 10 times as large as that obtained in Ce:Mn:LN.

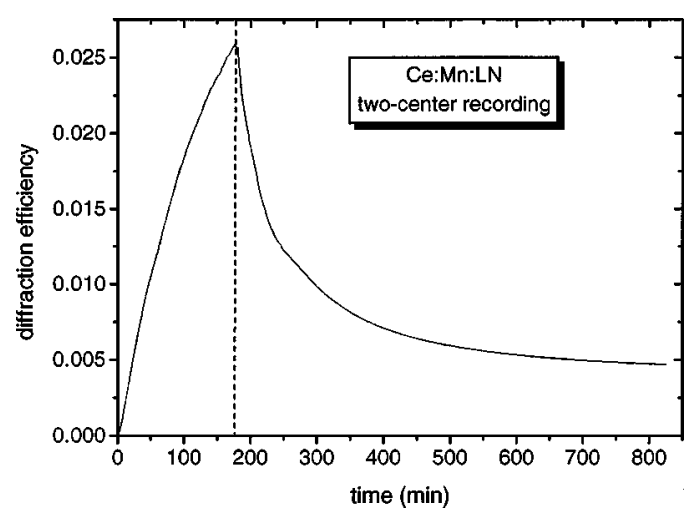

FIG. 4. Evolution of the diffraction efficiency during two-center holographic recording in $\mathrm{Ce}$ and $\mathrm{Mn}$ doubly doped $\mathrm{LiNbO}_{3}$. During recording, ultraviolet light illuminates the sample, while it is blocked during readout.

\section{Photovoltaic current}

Recording sensitivity and saturated diffraction efficiency depend on the photovoltaic constant. ${ }^{6,15}$ During homogeneous illumination, the short-circuited photocurrent density is proportional to the absorbed power density, i.e.,

$$
J=\kappa \alpha I,
$$

where $I$ is the incident intensity, $\alpha$ is the absorption coefficient, and $\kappa$ is the photovoltaic constant depending mainly on absorption center and wavelength. For the thick samples, the light depletion due to absorption must be considered and an averaged light intensity $\bar{I}=I[1-\exp (-\alpha d)](\alpha d)^{-1}$ must be used.

Measurements of the photocurrents are made with an electrometer having an input impedance much less than the crystal impedance. For illumination, we use a diode-pumped frequency-doubled Nd:YAG laser (wavelength $532 \mathrm{~nm}$ and power $400 \mathrm{~mW}$ ). The ordinarily polarized beam is expanded to illuminate the whole sample homogeneously. All measurements are made at room temperature $\left(25^{\circ} \mathrm{C}\right)$. After the illumination beam illuminates the sample for at least $10 \mathrm{~min}$, the steady-state photocurrent is detected. The delay is required to eliminate the influence of pyroelectric currents. ${ }^{16}$ The determined photovoltaic constant for reduced Fe:LN is $\kappa=1.3 \times 10^{-9} \mathrm{~cm} / \mathrm{V}$ at $532 \mathrm{~nm}$, which is in good agreement with the results reported by Krätzig and Kurz. ${ }^{6}$ For reduced Ce:LN, $\kappa=0.4 \times 10^{-9} \mathrm{~cm} / \mathrm{V}$ at $532 \mathrm{~nm}$ is measured. Ordinarily polarized red light $(633 \mathrm{~nm})$ is also employed to illuminate the samples. In the case of $\mathrm{Fe}: \mathrm{LN} \quad \kappa=0.8$ $\times 10^{-9} \mathrm{~cm} / \mathrm{V}$ is obtained, while the value of the photovoltaic constant in Ce:LN cannot be determined under the present experimental accuracy.

\section{E. Reflection grating}

In $\mathrm{LiNbO}_{3}$ crystals, bulk photovoltaic currents as well as diffusion currents contribute to formation of the spacecharge field during holographic recording. With a decrease of the grating spacing, the diffusion field becomes more important. ${ }^{17}$ The dynamics of the formation of the reflection grating can be used for detection of diffusion-related properties.

In our experiment, two ordinarily polarized beams impinge on the two opposite $c$ faces of the Ce:LN crystal, with an angle of incidence of about $5^{\circ}$ in air. The length of the sample along the $c$ axis is $7 \mathrm{~mm}$. The diffraction efficiency as a function of recording time is monitored. For the sake of comparison with the performance of transmission gratings, we convert the efficiency into the modulation of refractive index by taking reflection into account using the following relations: ${ }^{18}$

$$
\eta=R \sin ^{2}\left(\frac{\pi \Delta n d}{\lambda \cos \theta_{i}}\right)
$$

for the transmission grating and

$$
\eta=R \tanh ^{2}\left(\frac{\pi \Delta n d}{\lambda \cos \theta_{i}}\right)
$$




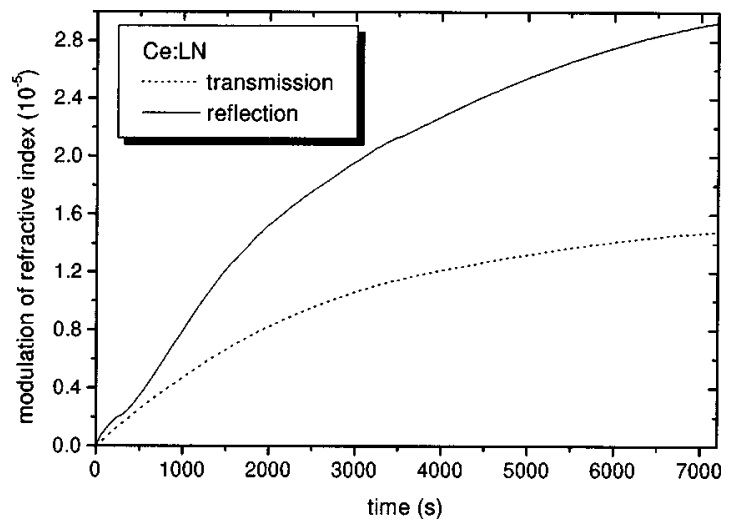

FIG. 5. Modulation of the refractive-index as a function of recording time for reflection geometry (solid curve) and transmission geometry (dotted curve) in Ce:LN. Wavelength and intensity of writing beam are $633 \mathrm{~nm}$ and $26 \mathrm{~mW} / \mathrm{cm}^{2}$, respectively. In transmission geometry both beams impinge on the $b$ face with an angle of $23^{\circ}$, while in reflection geometry both beams impinge on the two opposite $c$ faces with angle $5^{\circ}$ in air. The grating vector of the interference pattern is in both cases aligned along the crystallographic $c$ axis.

for the reflection grating. In the above equations, $R$ is the factor caused by reflection loss, $\theta_{i}$ is the angle of incidence inside the crystal, $d$ is the thickness along the grating vector, and the absorption is not considered due to the small value at red. As shown in Fig. 5, recording in the reflection geometry is faster than that in the transmission geometry. The corresponding sensitivity in the case of reflection geometry is $S$ $=0.009 \mathrm{~cm} / \mathrm{J}$. The modulation of refractive index in the reflection geometry is twice as large as that in the transmission grating for Ce:LN. In Fe:LN, however, the reflection grating is only 1.4 times stronger than the transmission grating.

\section{DISCUSSION}

The experimental results described above clearly show that $\mathrm{Ce}$ is a photorefractive dopand for $\mathrm{LiNbO}_{3}$. An increase of the Ce concentration leads to a substantial increase of the sensitivity in the red spectral region. It is very interesting that the absorption change in Ce:Mn:LN with the aid of ultraviolet illumination is larger than that in the Fe:Mn:LN. Because red light essentially cannot excite electrons from Mn traps, the absorption of doubly doped $\mathrm{LiNbO}_{3}$ for red light is proportional to the density of the filled shallower traps ( $\mathrm{Fe}$ or $\mathrm{Ce})$. Anyhow, the recording sensitivity in Ce singly doped $\mathrm{LiNbO}_{3}$ is smaller than that in the Fe-doped one even through the concentration of $\mathrm{Ce}$ ions is twice as large as that of $\mathrm{Fe}$ ions. We know that in $\mathrm{LiNbO}_{3}$ both the sensitivity and the maximum diffraction efficiency depend on the photovoltaic constant. So the smaller sensitivity in the Ce-doped sample can be attributed to the much smaller photovoltaic constant. Photovoltaic constant can be expressed as ${ }^{19}$

$$
\kappa=\left(e / h \nu_{0}\right) \Phi L,
$$

where $h \nu_{0}$ is the photon energy, $\Phi$ is the quantum efficiency that an absorbed photon will produce in a photoionized charge, and $L$ is the migration length of an electron in the conduction band before it is recaptured. From the results obtained with light of $532 \mathrm{~nm}$, we get $\Phi L=3 \times 10^{-11} \mathrm{~m}$ for the Fe-doped sample, while $\Phi L$ is approximately 1 $\times 10^{-11} \mathrm{~m}$ for the Ce-doped sample.

The amplitude of the steady-state space-charge field $E_{\text {sc }}$ in photorefractive materials is determined by ${ }^{20}$

$$
E_{\mathrm{sc}}=\left[\frac{E_{P}^{2}+E_{D}^{2}}{\left(1+E_{D} / E_{q}\right)^{2}+\left(E_{p} / E_{q}^{\prime}\right)^{2}}\right]^{1 / 2},
$$

where $E_{P}$ is the photovoltaic field which is proportional to the ionized trap density, $E_{D}$ is the diffusion field which depends linearly on the amplitude of the grating vector, and $E_{q}$ and $E^{\prime}{ }_{q}$ are limiting space-charge fields determined by effective trap density and filled trap density, respectively. In the case of oxidized $\mathrm{Fe}: \mathrm{LiNbO}_{3}$ where the density of emptied Fe centers is much larger than that of filled centers, the saturated refractive index is inversely proportional to the grating spacing. This means that a reflection grating should have a smaller amplitude compared to that of a transmission grating. ${ }^{21}$ But the sample we used in the reflection geometry is strongly reduced. If we suppose that no limitation of space-charge fields is present, Eq. (5) simplifies to

$$
E_{\mathrm{sc}}=\left(E_{P}^{2}+E_{D}^{2}\right)^{1 / 2} \text {. }
$$

We know that $E_{P}$ is the same in both transmission and reflection geometry. However, $E_{D}$ is larger in reflection geometry. This is due to the fact that $E_{D}$ is inversely proportional to the grating spacing. Therefore, we expect to obtain a larger $E_{\mathrm{sc}}$. The enhancement of performance for the $\mathrm{Fe}-$ doped sample in the reflection geometry is not so large. This can be attributed to a much higher photovoltaic field, which is independent of the amplitude of the grating wave vector.

In conclusion, $\mathrm{Ce}$ is an active red-sensitive photorefractive center in $\mathrm{LiNbO}_{3}$ and can play an important role in charge transport during holographic recording. However, the photovoltaic constant in Ce-doped crystals is smaller than that in Fe-doped ones. This makes the recording in Ce-doped crystals not as sensitive as that in Fe-doped crystals. In reflection geometry, where diffusion field makes more contribution, the photorefractive performance of Ce-doped samples is enhanced.

\section{ACKNOWLEDGMENTS}

This work was supported by National Science Foundation Center for Neurmorphic Systems Engineering at the California Institute of Technology. The authors thank Yunping Yang for helpful discussion.

${ }^{1}$ D. Psaltis and F. Mok, Sci. Am. 273, 70 (1995).

${ }^{2}$ J. F. Heanue, M. Bashaw, and L. Hesselink, Science 265, 749 (1994).

${ }^{3}$ M. P. Bernal et al. Appl. Opt. 35, 2360 (1996).

${ }^{4}$ I. McMichael, W. Christian, D. Pletcher, T. Y. Chang, and J. H. Hong, Appl. Opt. 35, 2375 (1996).

${ }^{5}$ W. Phillips and D. L. S. J. J. Amodei, RCA Rev. 33, 94 (1972).

${ }^{6}$ E. Krätzig and H. Kurz, Opt. Acta 24, 475 (1977).

${ }^{7}$ K. Buse, A. Adibi, and D. Psaltis, Nature (London) 393, 665 (1998).

${ }^{8}$ K. Megumi, H. Kozuka, M. Kobayashi, and Y. Furuhta, Appl. Phys. B: Photophys. Laser Chem. 30, 631 (1977).

${ }^{9}$ C. Yang, Y. Zhang, P. Yeh, and X. Wu, Opt. Commun. 113, 416 (1995).

${ }^{10}$ S. Dou, Y. Lian, H. Gao, Y. Zhu, X. Wu, C. Yang, and P. Ye, Appl. Opt. 34, 2024 (1995)

${ }^{11}$ K. Xu, H. Xu, Y. Yuan, J. Hong, and Y. Xu, Proc. SPIE 1078, 331 (1989). 
${ }^{12}$ S. Yin and F. T. S. Yu, IEEE Photonics Technol. Lett. 5, 581 (1993).

${ }^{13}$ D. K. McMillen, T. D. Hudson, J. Wagner, and J. Singleton, Opt. Express 2, 491 (1998).

${ }^{14}$ K. Buse, Appl. Phys. B: Lasers Opt. 64, 273 (1997).

${ }^{15}$ A. M. Glass, D. von der Linde, and T. J. Negran, Appl. Phys. Lett. 25, 233 (1974).

${ }^{16}$ X. Yue, S. Mendricks, T. Nikolajsen, D. Kip, H. Hesse, and E. Krätzig, J. Opt. Soc. Am. B 16, 389 (1999).
${ }^{17}$ A. Krumins, Z. Chen, and T. Shiosaki, Opt. Commun. 117, 147 (1995).

${ }^{18}$ H. Kogelnik, Bell Syst. Tech. J. 48, 2909 (1969).

${ }^{19}$ R. Grousson, M. Henry, S. Mallick, and S. L. Xu, J. Appl. Phys. 54, 3012 (1983).

${ }^{20}$ N. V. Kukhtarev, V. B. Markov, S. Odoulov, M. S. Soskin, and V. L. Vinetskii, Ferroelectrics 22, 949 (1979).

${ }^{21}$ K. Peithmann, A. Wiebrock, and K. Buse, Appl. Phys. B: Lasers Opt. 68, 777 (1999). 